\title{
Analyses of the efficacy of percutaneous kyphoplasty and alendronate sodium on thoracolumbar vertebral fracture and the risk factors of fracture
}

\author{
PENGBIN LI $^{1 *}$, WENJUAN TANG ${ }^{2 *}$, BIAO CHE $^{1}, \mathrm{KAI} \mathrm{WANG}^{1}, \mathrm{KAI} \mathrm{ZOU}^{1}$, \\ ZHENG JIN ${ }^{3}$, FENG ZHOU ${ }^{3}$ and YAN ZHUANG ${ }^{3 *}$ \\ ${ }^{1}$ Department of Orthopedics, General Hospital of The Yangtze River Shipping and Wuhan Brain Hospital; \\ ${ }^{2}$ Department of Radiology, Union Hospital, Tongji Medical College, Huazhong University of Science and Technology, \\ Wuhan, Hubei 430010; ${ }^{3}$ Department of Orthopedics, The Affiliated Jiangyin Hospital of \\ Southeast University Medical College, Wuxi, Jiangsu 214400, P.R. China
}

Received November 16, 2017; Accepted May 29, 2018

DOI: $10.3892 /$ etm.2018.6247

\begin{abstract}
The present study investigated the efficacy of percutaneous kyphoplasty and alendronate sodium on thoracolumbar vertebral fracture, and the risk factors leading to the recurrence of fracture. In the present study, a total of 80 patients with thoracolumbar vertebral fracture who were admitted to the Affiliated Jiangyin Hospital of Southeast University Medical College between January 2014 and March 2016 for combination treatment of percutaneous kyphoplasty and alendronate sodium were enrolled. According to the recurrence of fracture, the patients were divided into two groups, the observation group (patients with fracture recurrence, $n=40$ ) and control group (patients with no fracture recurrence, $n=40$ ). All patients participated in a 1-year follow-up. The recurrence of fracture and the site of fracture were identified through the clinical symptoms and examination of the spine using magnetic resonance imaging. In addition, comparisons of the time of alleviation in numbness of lower limb and that in pains in waist and legs were carried out. Furthermore, statistics on the adverse reactions during intervention in the two groups were also collected; changes in visual analogue scale (VAS) and Oswestry Disability Index (ODI) of pains at different time points in two groups were also observed. One-way analysis and multivariate analysis were performed to identify the relevant risk factors. Alleviation time in numbness of lower legs in patients of the control group was significantly earlier than that in the observation group $(\mathrm{P}<0.05)$ and the alleviation
\end{abstract}

Correspondence to: Dr Yan Zhuang, Department of Orthopedics, The Affiliated Jiangyin Hospital of Southeast University Medical College, 163 Shoushan Road, Jiangyin, Wuxi, Jiangsu 214400, P.R. China

E-mail: zhuangyan_bmu@126.com

*Contributed equally

Key words: percutaneous kyphoplasty, alendronate sodium, thoracolumbar vertebral fracture, risk factor time in pains of the waist and legs of patients in the control group was also significantly earlier than that in the observation group $(\mathrm{P}<0.05)$. Furthermore, the incidence rates of abdominal pain, diarrhea, constipation and hypocalcemiain in the control group were also significantly lower compared with those in the observation group $(\mathrm{P}<0.05)$. One week, one month and one year after operation, the scores of VAS of pains and ODI in the control group were significantly lower compared with those in the observation group in the same period $(\mathrm{P}<0.05)$. Lower preoperative bone density and exosmosis of bone cement in treatment were the independent risk factors leading to the recurrence of fracture. For patients with thoracolumbar vertebral fracture who received the combination treatment of percutaneous kyphoplasty and alendronate sodium, there underlies an important correlation between the recurrence rate of fracture and the preoperative bone density as well as the exosmosis of bone cement in operation.

\section{Introduction}

With the aged tendency of population and industrialization in China, the proportion of complications like osteoporosis is increasing. In addition to the risk factors in traffic and work, the proportion in thoracolumbar vertebral fracture is also increasing (1). Thoracolumbar vertebral fracture patients are usually complicated with severe and persistent acute pains in waist and back, dysfunction in nerve system (2), or even progressive deformity in waist and back with the progression of disease (3). In clinical practices, surgical treatment is preferred (4). However, open surgery is more suitable for patients complicated with spinal cord dysfunction due to the massive trauma (5), poor tolerance and slow postoperative recovery of patients, while the micro-invasive surgery can recover the spinal cord function rapidly with less pain and correction of deformity (6). Among these methods, percutaneous kyphoplasty is a more frequent micro-invasive surgical method in clinical practices so far (7).

Alendronate sodium, as one of the most common regulators in bone metabolism in clinical practices (8), manifests a 
strong affinity with the intraosseous hydroxyapatite, which enables it to inhibit the activity of osteoclast, thereby inducing the physiological effect of osteoblast to suppress the bone absorption (9) and facilitating the bone mineralization and deposition (10). Recurrence rate of fracture remains high in some patients who undergo the percutaneous kyphoplasty, especially those complicated with osteoporosis, despite of its various advantages, which should be focused on in clinical research and treatment (11). In this study, the analysis was carried out mainly for the efficacy of percutaneous kyphoplasty in combination with alendronate sodium on thoracolumbar vertebral fracture, as well as the risk factors leading to the recurrence of fracture after surgery.

\section{Patients and methods}

General data. A total of 80 patients with thoracolumbar vertebral fracture who were admitted to were admitted to General Hospital of The Yangtze River Shipping and Wuhan Brain Hospital and The Affiliated Jiangyin Hospital of Southeast University Medical College (Wuxi, China) between January 2014 and March 2016 for combination treatment of percutaneous kyphoplasty and alendronate sodium were enrolled. Before operation, all patients aged below 60 years old were required to undergo computed tomography (CT) and magnetic resonance imaging (MRI) (Fig. 1A and B) for diagnosis to rule out those complicated with tuberculosis of thoracolumbar spine, tumors in thoracolumbar and vertebra, coagulation disorders, dysfunctions in lumbar vertebra, heart, lung, liver or kidney, diseases in spine or nervous system, mental diseases, diseases in consuming system, lateral displacement or rotation in fracture site, complete spinal cord injury after fracture or cauda equina syndrome. In addition, all patients had signed the written informed consent, and the protocols of this study had been approved by the Ethic's Committee of General Hospital of The Yangtze River Shipping and Wuhan Brain Hospital and The Affiliated Jiangyin Hospital of Southeast University Medical College.

According to the recurrence of fracture, all patients were divided into two groups, observation group (patients with fracture recurrence, $n=40$ ) and control group (patients with no fracture recurrence, $n=40$ ). In the observation group, there were 30 males and 10 females aged between 18 and 60 years old with an average age of 43.2 \pm 2.1 years old; as for the fracture causes, there were 25 cases caused by traffic accident, 13 by high-altitude falling accident and 2 by bruise; in terms of the Frankel classification of damage to spinal cord, there were 5 of class B, 15 of class C, 10 of class D and 10 of class E; for the site of fracture, there were 13 in thoracic vertebra, 19 in lumbar vertebra and 8 in thoracolumbar vertebra; the time from onset of fracture to surgery ranged from 8 to $48 \mathrm{~h}$ with an average of $18.3 \pm 2.1 \mathrm{~h}$. In the control group, there were 31 males and 9 females aged between 18 and 60 years old with an average age of $44.1 \pm 2.0$ years old; as for the fracture causes, there were 26 cases caused by traffic accident, 12 by high-altitude falling accident and 2 by bruise; in terms of the Frankel classification of damage to spinal cord, there were 6 of class B, 16 of class C, 9 of class D and 9 of class E; for the site of fracture, there were 12 in thoracic vertebra, 15 in lumbar vertebra and 10 in thoracolumbar vertebra; the time from onset of fracture
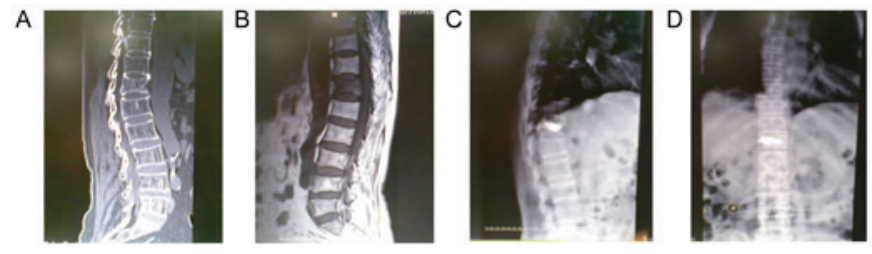

Figure 1. Pre- and post-operative images. (A) Pre-operative CT images (B) Pre-operative magnetic resonance imaging images. (C and D) Post-operative $\mathrm{X}$-ray images.

to surgery ranged from 8 to $48 \mathrm{~h}$ with an average of $18.4 \pm 2.0 \mathrm{~h}$. Comparisons of the gender, age, causes of fracture, degree of damage to spinal cord, site of fracture and the time from onset of fracture to surgery showed that the differences had no statistical significance $(\mathrm{P}>0.05)$.

Treatment methods. Under local anesthesia, all patients underwent percutaneous kyphoplasty, and during the surgery, patients were required to keep in prone with the hip and anterior superior spine being lifted to keep the abdomen suspended for identify the fracture site of vertebra under X-ray. From the pedicle of vertebral arch in the affected vertebra under local anesthesia, locating pin was guided into the $1 / 3$ site of vertebra, and, after the pathway was expanded manually by a drill, reached to the site $1 \mathrm{~cm}$ to the anterior edge of vertebra; again, X-ray examination was then performed to guarantee that the tip of pin was in the midline of vertebra. Thereafter, a saccule was inserted with the pressure being adjusted to 160 to $180 \mathrm{psi}$, and the vertebra was then slowly opened for injection of about $1 \mathrm{ml}$ of bone cement under X-ray, after which the distribution of bone cement should be closely monitored. At $60 \mathrm{sec}$ later, approximately $1 \mathrm{ml}$ of bone cement was again injected, and the distribution range of bone cement should also be closely monitored to avoid the exosmosis of bone cement to the posterior edge veins of vertebra. In this surgery, the amount of bone cement injection was recorded until the bone cement was coagulated, and surgery was completed after patients kept the prone position for $20 \mathrm{~min}$. After surgery, $10 \mathrm{mg}$ alendronate sodium (SFDA approval no. H10980108; CSPC Huasheng Pharmaceutical Co., Ltd., Shijiazhuang, China) was administrated orally in the morning before meal once per day. Additionally, post-operation X-ray of all patients were done to observe the effections of the operation (Fig. 1C and D). All patients underwent 1-year follow-up. Recurrence of fracture and the site were identified through the clinical symptoms and the results of MRI of spine cord.

Observation indexes and evaluation methods. The alleviation time of numbness in lower limbs and pains in waist and legs in two groups were compared. Statistics on the adverse reaction during intervention in two groups were collected. visual analogue scale (VAS) and Oswestry Disability Index (ODI) scores of pains at different time points in two groups were compared, and comparisons of age, section of fracture and surgery, preoperative bone density, preoperative kyphotic angle, preoperative height of anterior edge of affected vertebra and amount of bone cement in operation were also performed. One-way and multivariate analyses were performed for data above to identify the relevant risk factors. As for the pain 
Table I. Comparison of the alleviation time of numbness in lower limbs and pains in waist and legs between two groups after surgery $(\mathrm{d}$, mean \pm standard deviation).

\begin{tabular}{lcc}
\hline Variable & Alleviation time in numbness of lower limbs & Alleviation time in pains in waist and legs \\
\hline Control group & $1.3 \pm 0.1$ & $1.6 \pm 0.3$ \\
Observation group & $3.6 \pm 0.3$ & $2.7 \pm 0.5$ \\
$\mathrm{t}$ & 46.000 & 11.931 \\
P-value & $<0.001$ & $<0.001$ \\
\hline
\end{tabular}

evaluation, VAS scale was adopted, (10 points for intolerable pains, and 0 point for no pain), and a higher score meant that the pain was more acute. For ODI, the score was set from 0 to 45 points, and a higher score meant that the dysfunction was more evident.

Statistical analysis. SPSS 21.0 software (IBM Corp., Armonk, NY, USA) was adopted in this study. Measurement data were presented as mean \pm standard deviation, and t-test was carried out for mean comparison between two groups. Chi-square test was performed for rate comparison between two groups. As for the factors of patients, like age, sections of fracture and surgery, preoperative bone density, preoperative kyphotic angle, preoperative height of anterior edge of affected vertebra and amount of bone cement in operation, one-way ANOVA test followed by post hoc test (Least Significant Difference) was firstly performed, and for those with statistical significance, non-conditional multivariate Logistic regression analysis was adopted. $\mathrm{P}<0.05$ was considered to indicate a statistically significant difference.

\section{Results}

Comparison of the alleviation time of numbness in lower limbs and pains in waist and legs between two groups after surgery. The alleviation time in numbness of lower limbs in patients of the control group was earlier than that in the observation group $(\mathrm{P}<0.05)$, and the differences are statistically significant. The alleviation time in the waist and legs of patients in the control group was also earlier than that in the observation group, and the differences are statistically significant $(\mathrm{P}<0.05$; Table I). It suggested that among patients in the observation group after the first time of surgery, the alleviation time of numbness in lower limbs and pains in waist and legs after the first time of surgery is more significantly retarded than those in the control group.

Comparisons of the adverse reactions during intervention between two groups. The incidence rates of abdominal pain, diarrhea, constipation and hypocalcemia in the control group were also lower than those in the observation group, and the differences are statistically significant $(\mathrm{P}<0.05$; Table II $)$. It suggested that complications like abdominal pains, diarrhea, constipation and hypocalcemia occur seldom in patients without fracture recurrence.

Comparison of the changes in VAS scores of pains at different time points between two groups. One week, one month and even one year after operation, the scores of VAS of pains in the control group were $3.2 \pm 0.3$ points, $2.6 \pm 0.2$ points and $1.5 \pm 0.1$ points, significantly lower than those of the recurrence patients $(4.3 \pm 0.3$ points, $3.3 \pm 0.3$ points and $2.5 \pm 0.3$ points $)$ in the observation group in the same period $(\mathrm{t}=16.398,12.279$ and 20.000; $\mathrm{P}<0.05$; Fig. 2). It revealed that patients with fracture recurrence after percutaneous kyphoplasty have a much higher pain score.

Comparison of the changes in ODI scores of pains at different time points between two groups. One week, one month and even one year after operation, the scores of ODI in the control group were $23.3 \pm 1.8$ points, $15.7 \pm 1.2$ points and $10.8 \pm 0.8$ points, significantly lower than those ( $30.2 \pm 2.5$ points, $19.6 \pm 1.8$ points and $13.7 \pm 0.9$ points) in the observation group in the same period $(\mathrm{t}=14.166,11.402$ and $15.232 ; \mathrm{P}<0.05 ;$ Fig. 3$)$. It revealed that patients with fracture recurrence after percutaneous kyphoplasty have a relatively lower score of ODI.

Comparisons of the results of one-way analysis of measurement data between two groups. Through one-way analysis, it was found that the amount of fracture and surgery sections, preoperative bone density, preoperative kyphotic angle, preoperative height of anterior edge of affected vertebra, amount of bone cement in operation, onset time and exosmosis of bone cement were the relevant risk factors for the recurrence of fracture after the percutaneous kyphoplasty in combination with alendronate sodium for patients with thoracolumbar vertebral fracture (Tables III and IV).

Multivariate logistic regression analysis of the recurrence of fracture. With the recurrence of fracture as the dependent variable, the multivariate logistic regression analysis was performed and the results revealed that a lower preoperative bone density and the exosmosis of bone cement during surgery were the independent risk factors in the recurrence of fracture (Table V).

\section{Discussion}

After treatment of percutaneous kyphoplasty in combination with alendronate sodium for thoracolumbar vertebral fracture, some patients may suffer from the recurrence of fracture (12), which usually leads to the severe pains in waist and back, progressive collapse of spinal cord, kyphosis osteotomy of vertebral laminae or even dysfunction in spinal cord, thereby affecting the normal motor function (13). Recurrence not only decreases the life quality of patients, but also brings heavy 
Table II. Comparisons of the adverse reactions during intervention between two groups (n, \%).

\begin{tabular}{lcccc}
\hline Variable & $\begin{array}{c}\text { Abdominal pains } \\
\text { and diarrhea }\end{array}$ & Constipation & Hypocalcemia & Total incidence rate \\
\hline Control group & 1 & 0 & 1 & $2(5.0 \%)$ \\
Observation group & 3 & 4 & 3 & $10(25.0 \%)$ \\
$\chi^{2}$ & & - & 4.804 \\
P-value & & - & 0.028 \\
\hline
\end{tabular}

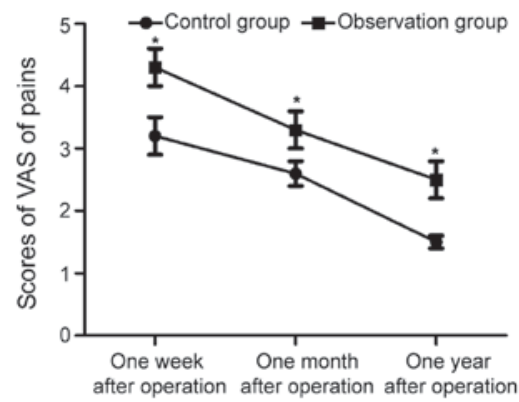

Figure 2. Comparison of the changes in VAS scores of pains at different time points between the two groups. One week, one month and one year after operation, the VAS scores of the pains in the control group were significantly lower compared with those in the observation group in the same time period. ${ }^{*} \mathrm{P}<0.05$ vs. Control group. VAS, visual analogue scale.

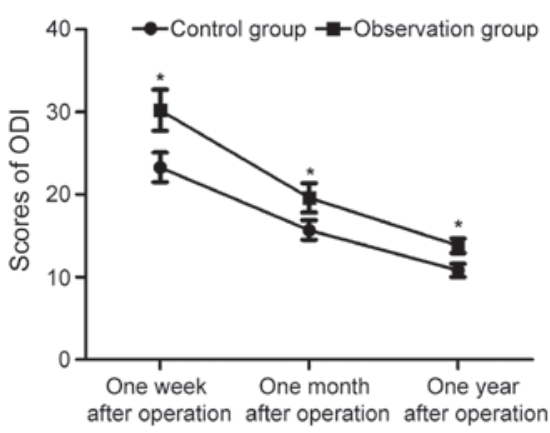

Figure 3. Comparison of the changes in ODI scores of pains at different time points between the two groups. One week, one month and one year after operation, the ODI scores in the control group were significantly lower compared with those in the observation group in the same period. ${ }^{*} \mathrm{P}<0.05$ vs. Control group. ODI, Oswestry Disability Index.

burden for the society and the family of patients (14). A previous study (15) believed that osteoporosis is the most common cause for recurrence of fracture in percutaneous kyphoplasty. But, there remain few studies currently focusing on the causes responsible for the recurrence of fracture after administration of alendronate sodium for symptomatic treatment. We suspected that there were important relationships between the recurrence of fracture and quantity of fracture, surgery sections, preoperative bone density, preoperative kyphotic angle, preoperative height of anterior edge of affected vertebra, intraoperative usage amount of bone cement, onset time of fracture and exosmosis of bone cement after administration of alendronate sodium.

In this study, patients with thoracolumbar vertebral fracture who received the percutaneous kyphoplasty in combination with alendronate sodium were enrolled to figure out the recurrence of fracture. It was found that in comparison of the alleviation time of numbness in lower limbs and pains in waist and legs between two groups after treatment, the alleviation time of numbness in lower limbs in the control group was significantly earlier than that in the observation group, and the alleviation time of pains in waist and legs in the control group was also earlier than that in the observation group, suggesting that among patients in the observation group after the first time of surgery, the alleviation time of numbness in lower limbs and pains in waist and legs after the first time of surgery is more significantly retarded than those in the control group. Meanwhile, comparison of incidence rate of adverse reaction during intervention between two groups also showed that the total incidence rates of abdominal pains, diarrhea, constipation and hypocalcemia in the patients of the control group were significantly lower than those in the observation group, suggesting that complications like abdominal pains, diarrhea, constipation and hypocalcemia occur seldom in patients without fracture recurrence, which may be correlated with the absorption after treatment of alendronate sodium. In addition, the analyses in changes of VAS scores of pains and ODI scores at different time points in two groups showed that one week, one month and even one year after operation, the scores of VAS of pains and ODI in the control group were significantly lower than those in the observation group in the same period, revealing that patients with fracture recurrence after percutaneous kyphoplasty have a much higher pain score and a relatively lower score of ODI. Finally, one-way analysis and multivariate analysis for relevant risk factors leading to the fracture recurrence after percutaneous kyphoplasty in combination with alendronate sodium showed that a large quantity of fracture and surgery sections, preoperative bone density, preoperative kyphotic angle, preoperative height of anterior edge of affected vertebra, intraoperative usage amount of bone cement, onset time of fracture and exosmosis of bone cement are the relevant risk factors leading to the fracture recurrence after percutaneous kyphoplasty in combination with alendronate sodium, and the low preoperative bone density and exosmosis of bone cement in surgery are also the independent risk factors of fracture recurrence.

Among the patients with fracture recurrence after percutaneous kyphoplasty in combination with alendronate sodium for thoracolumbar vertebral fracture, elder patients with a relatively low bone density are the dominant population (16). The reason may be that bone mineral density decreased significantly with the growth of the age, especially in older postmenopausal women. In the present study, bone density is 
Table III. Comparisons of the results of one-way analysis of measurement data between two groups (mean \pm standard deviation).

\begin{tabular}{lcrrr}
\hline Variable & Observation group & Control group & $\mathrm{t}$ & P-value \\
\hline Age (years old) & $65.7 \pm 2.1$ & $66.0 \pm 2.1$ & 0.639 & 0.525 \\
Section of fracture (n) & $2.15 \pm 0.03$ & $1.36 \pm 0.02$ & 138.575 & $<0.001$ \\
Section of surgery (n) & $2.03 \pm 0.03$ & $1.12 \pm 0.02$ & 159.625 & $<0.001$ \\
Preoperative bone density (standard deviation) & $-3.24 \pm 0.12$ & $-2.79 \pm 0.09$ & 18.974 & $<0.001$ \\
Preoperative kyphotic angle $\left(^{\circ}\right)$ & $23.56 \pm 0.21$ & $19.50 \pm 0.18$ & 92.838 & $<0.001$ \\
Preoperative height of anterior edge of affected vertebra (mm) & $0.59 \pm 0.03$ & $0.65 \pm 0.04$ & 7.589 & $<0.001$ \\
Amount of bone cement in operation (ml) & $3.21 \pm 0.08$ & $3.56 \pm 0.09$ & 18.383 & $<0.001$ \\
\end{tabular}

Table IV. Comparisons of the results of one-way analysis of enumeration data between two groups (n).

\begin{tabular}{|c|c|c|c|c|}
\hline Variable & Observation group & Control group & $\chi^{2}$ & P-value \\
\hline Sex & & & & 0.805 \\
\hline Male & 11 & 12 & 0.061 & \\
\hline Female & 29 & 28 & & \\
\hline Time of fracture & & & & 0.008 \\
\hline Within 1 month & 28 & 38 & 7.013 & \\
\hline Over 1 month & 12 & 2 & & \\
\hline Hormone administration & & & & 0.302 \\
\hline Yes & 12 & 8 & 1.067 & \\
\hline No & 28 & 32 & & \\
\hline Exosmosis of bone cement & & & & $<0.001$ \\
\hline Yes & 20 & 2 & 18.119 & \\
\hline No & 20 & 38 & & \\
\hline
\end{tabular}

Table V. Multivariate logistic regression analysis of fracture recurrence.

\begin{tabular}{|c|c|c|c|c|c|c|}
\hline Variable & $\beta$ & $\begin{array}{l}\text { Standard } \\
\text { error }\end{array}$ & Weight & P-value & $\begin{array}{l}\text { Odds } \\
\text { ratio }\end{array}$ & $\begin{array}{l}95 \% \text { confident } \\
\text { interval }\end{array}$ \\
\hline Fracture section (n) & 0.786 & 0.613 & 1.645 & 0.201 & 2.193 & $0.661-7.729$ \\
\hline Surgery section (n) & 0.045 & 0.060 & 0.579 & 0.448 & 1.047 & $0.933-1.173$ \\
\hline Preoperative bone density (SD) & -1.835 & 0.777 & 5.595 & 0.018 & 0.159 & $0.035-0.731$ \\
\hline Preoperative kyphotic angle $\left({ }^{\circ}\right)$ & 0.035 & 0.044 & 0.601 & 0.439 & 1.035 & $0.951-1.127$ \\
\hline $\begin{array}{l}\text { Preoperative height of anterior } \\
\text { edge of affected vertebra (mm) }\end{array}$ & 0.223 & 0.343 & 0.425 & 0.516 & 1.251 & $0.640-2.446$ \\
\hline Intraoperative amount of bone cement $(\mathrm{ml})$ & 0.938 & 3.207 & 0.086 & 0.771 & 2.553 & $0.005-1368.589$ \\
\hline Onset of fracture & 0.073 & 0.053 & 0.031 & 0.856 & 2.693 & $0.491-2.361$ \\
\hline Bone cement exosmosis & 1.873 & 0.834 & 5.050 & 0.025 & 6.503 & $1.272-33.273$ \\
\hline
\end{tabular}

$\mathrm{SD}$, standard deviation. Bone density for normal subjects ranged from $-1 \mathrm{SD}$ to $+1 \mathrm{SD}$.

an independent risk factor affecting the fracture recurrence after surgery, suggesting that bone density is a major factor affecting the surgical efficacy, especially the recurrence of fracture $(17,18)$. In addition, exosmosis of bone cement is the most common complication in percutaneous kyphoplasty (19), and also the independent risk factor leading to the postoperative recurrence of fracture (20). In this study, it was found that for patients who undergo percutaneous kyphoplasty with or without administration of alendronate sodium for thoracolumbar vertebral fracture, the incidence rate of postoperative recurrence of fracture was significantly increased in patients complicated with the exosmosis of bone cement in surgery.

There are still some limitations in this study. Classifying the recurrence rate of fracture and the exosmosis of bone cement 
as 'Yes/No', we did not consider the type, severity and site of exosmosis, which could warrant the conclusions. Thus, further studies will be needed to clarify the relations of refracture types and severity and site of exosmosis. Additionally, the efficacy of alendronate sodium with PTH in treatment of Thoracolumbar Vertebral Fracture will also be explored in future.

In conclusion, our study indicated that percutaneous kyphoplasty combined with alendronate sodium was a superior treatment option of thoracolumbar vertebral fracture when compared with percutaneous kyphoplasty alone. Additionally, there underlies an important correlation between the recurrence rate of fracture and the preoperative bone density as well as the exosmosis of bone cement in operation for patients with thoracolumbar vertebral fracture who receive percutaneous kyphoplasty in combination with alendronate sodium.

\section{Acknowledgements}

Not applicable.

\section{Funding}

No funding was received.

\section{Availability of data and materials}

All data generated or analyzed during this study are included in this published article.

\section{Authors' contributions}

PL and WT designed the study, BC, KW, KZ and ZJ collected the data, FZ and YZ analyzed the data, PL and YZ prepared the manuscript, WT and $\mathrm{KZ}$ made the revisions, YZ and PL performed the operations. All authors read and approved the final manuscript.

\section{Ethics approval and consent to participate}

This study was approved by the Ethic Committee of General Hospital of The Yangtze River Shipping and Wuhan Brain Hospital and The Affiliated Jiangyin Hospital of Southeast University Medical College (Wuhan, China). Signed written informed consents were obtained from the patients and/or guardians.

\section{Patient consent for publication}

Not applicable.

\section{Competing interests}

The authors declare that they have no competing interests.

\section{References}

1. Semaan H, Obri T, Bazerbashi M, Paull D, Liu X, Sarrouj M and Elgafy $\mathrm{H}$ : Clinical outcome and subsequent sequelae of cement extravasation after percutaneous kyphoplasty and vertebroplasty: A comparative review. Acta Radiol: Jan1, 2017 (Epub ahead of print)
2. Niu J, Song D, Zhou H, Meng Q, Meng B and Yang H: Percutaneous kyphoplasty for the treatment of osteoporotic vertebral fractures with intravertebral fluid or air: A comparative study. Clin Spine Surg 30: 367-373, 2017.

3. Filippiadis DK, Marcia S, Masala S, Deschamps F and Kelekis A: Percutaneous vertebroplasty and kyphoplasty: Current status, new developments and old controversies. Cardiovasc Intervent Radiol 40: 1815-1823, 2017

4. Rajasekaran S, Kanna RM, Schnake KJ, Vaccaro AR, Schroeder GD, Sadiqi S and Oner C: Osteoporotic thoracolumbar Fractures-How are they different?-Classification and treatment algorithm. J Orthop Trauma 31 (Suppl 4): S49-S56, 2017.

5. Liu T, Li Z, Su Q and Hai Y: Cement leakage in osteoporotic vertebral compression fractures with cortical defect using high-viscosity bone cement during unilateral percutaneous kyphoplasty surgery. Medicine (Baltimore) 96: e7216, 2017.

6. Masoudi MS, Haghnegahdar A, Ghaffarpasand F and Ilami G: Functional recovery following early kyphoplasty versus conservative management in stable thoracuolumbar fractures in parachute jumpers: A randomized clinical trial. Clin Spine Surg 30: E1066-E1073, 2017.

7. Yang P, Lin J, Liu H, Shen H and Yang HL: Primary bone mantle cell lymphomas with multiple vertebral compression fractures: A case report. Oncol Lett 13: 1288-1292, 2017.

8. Sun ZY, Li XF, Zhao H, Lin J, Qian ZL, Zhang ZM and Yang HL: Percutaneous balloon kyphoplasty in treatment of painful osteoporotic occult vertebral fracture: A retrospective study of 89 cases. Med Sci Monit 23: 1682-1690, 2017.

9. Rosenbaum BP, Kshettry VR, Kelly ML, Mroz TE and Weil RJ: Trends in inpatient vertebroplasty and kyphoplasty volume in the united states, 2005-2011: Assessing the impact of randomized controlled trials. Clin Spine Surg 30: E276-E282, 2017.

10. Lin D, Hao J, Li L, Wang L, Zhang H, Zou W and Lian K: Effect of bone cement volume fraction on adjacent vertebral fractures after unilateral percutaneous kyphoplasty. Clin Spine Surg 30: E270-E275, 2017.

11. Pesenti S, Graillon T, Mansouri N, Adetchessi T, Tropiano P, Blondel B and Fuentes S: Use of pedicle percutaneous cemented screws in the management of patients with poor bone stock. Neurochirurgie 62: 306-311, 2016 (In French).

12. Wu YS, Zhang H, Zheng WH, Feng ZH, Chen ZX and Lin Y: Hidden blood loss and the influential factors after percutaneous kyphoplasty surgery. Eur Spine J 26: 1878-1883, 2017.

13. Gonschorek O, Hauck S, Weiss T and Buhren V: Percutaneous vertebral augmentation in fragility fractures-indications and limitations. Eur J Trauma Emerg Surg 43: 9-17, 2017.

14. Beall DP, Parsons B and Burner S: Technical strategies and anatomic considerations for an extrapedicular modified inferior endplate access to thoracic and lumbar vertebral bodies. Pain Physician 19: 593-601, 2016.

15. Dmytriw AA, Talla K and Smith R: Percutaneous sacroplasty for the management of painful pathologic fracture in a multiple myeloma patient: Case report and review of the literature. Neuroradiol J 30: 80-83, 2017.

16. Cheng X, Long HQ, Xu JH, Huang YL and Li FB: Comparison of unilateral versus bilateral percutaneous kyphoplasty for the treatment of patients with osteoporosis vertebral compression fracture (OVCF): A systematic review and meta-analysis. Eur Spine J 25: 3439-3449, 2016.

17. Riva A, Togni S, Giacomelli L, Franceschi F, Eggenhoffner R, Feragalli B, Belcaro G, Cacchio M, Shu H and Dugall M: Effects of a curcumin-based supplementation in asymptomatic subjects with low bone density: A preliminary 24-week supplement study. Eur Rev Med Pharmacol Sci 21: 1684-1689, 2017.

18. Yang H, Liu H, Wang S, Wu K, Meng B and Liu T: Review of percutaneous kyphoplasty in china. Spine (Phila Pa 1976) 41 (Suppl 19): B52-B58, 2016.

19. Yaltirik K, Ashour AM, Reis CR, Ozdogan S and Atalay B: Vertebral augmentation by kyphoplasty and vertebroplasty: 8 years experience outcomes and complications. J Craniovertebr Junction Spine 7: 153-160, 2016.

20. Korovessis P, Mpountogianni E and Syrimpeis V: Percutaneous pedicle screw fixation plus kyphoplasty for thoracolumbar fractures A2, A3 and B2. Eur Spine J 26: 1492-1498, 2017.

This work is licensed under a Creative Commons Attribution-NonCommercial-NoDerivatives 4.0 International (CC BY-NC-ND 4.0) License. 